\title{
sciendo
}

\section{Early postnatal hypothalamic-pituitary-adrenal axis activity and reduced insulin sensitivity in adult rats}

\author{
Forouzan SAdeghimahalli ${ }^{1,2}$, Homeira Zardooz ${ }^{2}$, Ravieh GolCHOobian ${ }^{3}$ \\ ${ }^{1}$ Department of Physiology, School of Medicine, Mazandaran University of Medical Sciences, Sari, Iran; ${ }^{2}$ Neurophysiol- \\ ogy Research Center and Department of Physiology, Faculty of Medicine, Shahid Beheshti University of Medical Sciences, \\ Tehran, Iran; ${ }^{3}$ Cellular and Molecular Biology Research Center, Health Research Institute, Babol University of Medical \\ Sciences, Babol, Iran \\ E-mail:sadeghi.f.ph@gmail.com
}

Objective. Early life stress influences the development of metabolic disorders, including functional changes in the developing of pancreas mediated hypothalamic-pituitary-adrenal (HPA) axis. In the present study, the role of an early postnatal stress on corticosterone, glucose, and insulin levels was investigated during young adulthood.

Methods. Two groups of pups were studied, including control group (pups not receiving foot shock by communication box), and early stress group (pups receiving foot shock by communication box 2 times/day for 5 consecutive days). In rats, concentration of plasma corticosterone, glucose, and insulin was detected before and after placing them into the communication box at 2 weeks of age. At 8-10 weeks of age, concentrations of plasma corticosterone, glucose, and insulin and glucose tolerance were measured in young adult rats.

Results. Our results showed that early postnatal foot shock stress increased the corticosterone, insulin, and glucose levels in the postnatal age $(\mathrm{p}<0.01)$ that did not last until young adult age, but it caused a significant increase in plasma glucose and insulin levels $(\mathrm{p}<0.05)$ following the intraperitoneal glucose tolerance test (IPGTT) in young adult rats.

Conclusions. These results suggest that impaired IPGTT in young adult rats who experienced early postnatal stress can indicate insulin resistance or reduced insulin sensitivity that make it at risk of the type 2 diabetes later in life.

Key words: psychological stress, insulin sensitivity, adult, corticosterone, HPA axis

Stressful events at various life stages are risk factors for the development of many chronic diseases such as metabolic disorders. Stress exposure in the early postnatal period, as one of the critical stages of life for development, can induce permanent alterations in the hypothalamic-pituitary-adrenal (HPA) axis performance that leads to a malfunction in the developing organs (Taylor 2010; Maniam et al. 2014). Since the reorganization and development of the endocrine pancreatic tissue continue $2-3$ weeks and 3-4 years after birth in the rats and humans, respectively, pancreatic development may be impaired by early life stress. Therefore, the development of the pancreatic tissue may be impaired by stressful interventions in this time and in turn, it may lead to adverse effects on glucose homeostasis and insulin secretion, thus making the organism more susceptible to metabolic disorders and type-2 diabetes later in life (Hellerstrom and Swenne 1991; Bonner-Weir 2000; Hill et al. 2000; Fowden and Hill 2001; Srinivasan and Patel 2008; Jellyman et al. 2013). Several experimental and human studies have shown that early life stress may lead to a

Corresponding author: Forouzan Sadeghimahalli, Education Development Center, Mazandaran University of Medical Sciences, Sari, Iran; phone: +98 911 3263873; fax: +98 11 33373682; e-mail: sadeghi.f.ph@gmail.com. 
metabolic programming that increases the risk for the development of metabolic disorders later in life (Chida et al. 2007; Gluckman et al. 2008; Marais et al. 2008; Trombini et al. 2012; Zeugmann et al. 2012). A few studies have been done on the effect of early life stress on glucose homeostasis during puberty or adulthood. For example, maternal food insecurity stress did not change glucose and insulin concentrations, but glucose tolerance was impaired (Kaufman et al. 2007). In addition, maternal separation stress in mice increased the concentration of glucose, corticosterone, and adrenocorticotropic hormone (ACTH) in puberty, but insulin concentration remained unchanged (Loizzo et al. 2010). In another study, glucocorticoid overexposure in newborn foals increased the plasma insulin concentration after glucose tolerance test without any change in plasma glucose concentration during the glucose tolerance test, which is indicative of the pancreatic $\beta$-cells dysfunction (Jellyman et al. 2013). Regarding the possible relationship between postnatal stress and metabolic disorders, further investigations are needed to identify more risk factors for metabolic disorders and find the strategies that are likely to prevent the development and progression of them. Therefore, in the present study, the effect of an early postnatal foot shock stress on glucose tolerance during young adulthood was examined.

\section{Materials and methods}

Animals. Animals were kept in controlled environmental conditions $\left(20 \pm 2^{\circ} \mathrm{C}\right.$ and $12 \mathrm{~h}$ light-dark cycle) with free access to standard lab chow and water. Standard food produced by Pars Company (animal food producer, Iran) and tap water were provided throughout the experimental period.

Adult male and female Wistar rats were mated and then sixteen male rat pups were chosen from different litters for the study. Pups were divided into two control and the early stress groups. Pups at 2 weeks of age, in the early stress group, received foot shock stress 2 times/day for 5 consecutive days using a communication box. Animals in the control group at the same age were put into the communication box without exposure to foot shock stress 2 times/day for 5 consecutive days. In order to remove the effect of a novel environment, animals of two groups were placed in the communication box ( 2 times/day) for 5 days before the beginning of the experiments.

All experimental procedures were performed according to the Guide for the care and use of laboratory animals (National Institutes of Health Publication No. 80-23, revised 1996) and were approved by the Research and Ethics Committee of Shahid Beheshti University of Medical Sciences, Tehran, Iran.

Stress procedure. A communication box divided into nine compartments (each compartment: $21 \times 21 \times 19.5 \mathrm{~cm}$ ) was used to induce stress. The floor of five of the chambers were made from metal wire composed of stainless steel and connected to electricity; enabling the animals to receive foot shocks. An electric shock generator produced foot shocks $(0.8 \mathrm{~mA}, 1 \mathrm{~Hz})$ for $5 \mathrm{~s}$ at intervals of $60 \mathrm{~s}$ for $30 \mathrm{~min}$. In the early stress group, the rat pups received foot shock as mentioned above twice a day, between 10:00-12:00 and 13:00-15:00, for five consecutive days.

Blood sampling. Blood samples were taken from rats by cutting the tail before receiving the first foot shock and immediately after the last foot shock stress. In addition, blood samples obtained from adult rats at 10 weeks of age with the same procedures but in overnight fasting condition (16 hours). Before sampling, the animals were under sodium pentobarbital (Sigma, USA) anesthesia $60 \mathrm{mg} / \mathrm{kg}$; applied intraperitoneally (i.p.) (Sadeghimahalli et al. 2015). Blood samples were collected into heparinized tubes $(5000 \mathrm{IU} / \mathrm{ml})(5 \mu \mathrm{l} / \mathrm{ml}$ blood $)$ and centrifuged at $3000 \mathrm{~g}$ for $10 \mathrm{~min}$. Plasma was separated and kept at $-70^{\circ} \mathrm{C}$ for measuring the corticosterone, glucose, and insulin concentrations.

Measurement of plasma glucose, insulin, and corticosterone levels. Plasma samples were analyzed for insulin and corticosterone concentrations by the rat insulin ELISA kit (minimum detection: $0.07 \mu \mathrm{g} / \mathrm{l})$ (Mercodia, Sweden) and corticosterone ELISA kit (minimum detection: $1.631 \mathrm{nmol} / \mathrm{l}$ ) (DRG, Germany), respectively. Plasma glucose concentration was determined using the glucose oxidase method (minimum detection: $5 \mathrm{mg} / \mathrm{dl}$ ) (Pars Azmoon, Iran). Intra- and inter-assay coefficients of variations for insulin, corticosterone and glucose measurements were $2.2 \%$ and $3.9 \%, 6.6 \%$ and $7.24 \%$, and $2.8 \%$ and $2.19 \%$, respectively.

Intraperitoneal glucose tolerance test (IPGTT). Following overnight fasting, adult rats of two groups received under pentobarbital anesthesia an i.p. glucose ( $20 \%$ solution in water, $2 \mathrm{~g} / \mathrm{kg}$ b.w.) and then blood samples were taken after 10, 15, 30, 60, and 90 min in order to measure plasma glucose and insulin concentrations. Finally, linear and area under curves (AUC) were plotted (Sadeghimahalli et al. 2015).

Statistical analysis. All data were expressed as the mean \pm SEM. Unpaired t-test was performed by considering stress and/or glucose, insulin and corticosterone. For evaluating IPGTT, an analysis variance (ANOVA) with repeated measurement followed 
by Bonferroni test, was performed by Graph Pad Prism Version 5 program package (by considering time as a repeated factor and stress as an independent factor). P-values below 0.05 were considered to be statistically significant.

\section{Results}

The effect of postnatal stress on plasma corticosterone level in the neonatal rats. At 2 weeks of age, induction of foot shock stress for 5 consecutive days (2 times/day) increased plasma corticosterone significantly $(\mathrm{p}<0.01)$ in the early stress group as compared to the control group (Figure 1a).

The effect of postnatal stress on plasma glucose level in the neonatal rats. Plasma glucose significantly $(\mathrm{p}<0.01)$ enhanced following the induction of foot shock for 5 consecutive days ( 2 times/day) in the early stress group as compared to the control group (Figure 1b).

The effect of postnatal stress on plasma insulin level in the neonatal rats. In the Early stress group in comparison with the control group, the level of insulin plasma also increased significantly $(p<0.01)$ after using the foot shock stress for 5 consecutive days (2 times/day (Figure 1c).

The effect of postnatal stress on plasma corticosterone level in the young adult rats. At young adulthood, application of chronic postnatal foot shock stress did not alter the basal plasma corticosterone concentration in the early stress group as compared to the control group (Figure 2a).

The effect of postnatal stress on plasma glucose level in the young adult rats. Also, at young adulthood, the basal plasma glucose level in the early stress group following exposure to chronic postnatal foot shock stress did not change as compared to the control group (Figure 2b).

The effect of postnatal stress on plasma insulin level in the young adult rats. Using chronic postnatal foot shock at 2 weeks of age could increase the basal insulin plasma level non-significantly in the early stress group in comparison with the control group at young adulthood (Figure 2c).

The effect of postnatal stress on the IPGTT in the young adult rats. The IPGTT was performed to investigate the effect of postnatal stress on insulin resistance in young adult rats. Our results showed that during the IPGTT, plasma glucose levels increased significantly $(\mathrm{p}<0.05)$ at $30 \mathrm{~min}$ after the glucose injection in the early stress group as compared to the control group (Figure 3a). Plasma insulin concentrations in the early stress group were significantly higher than those of the control group at $30 \mathrm{~min}$ after glucose injection (Figure 3b). Also, the AUC obtained from calculating the surface under the glucose tolerance curve for plasma glucose was significantly higher, while AUC of insulin concentrations did not show significant differences between the early stress and the control groups (Figures $3 c$ and $3 \mathrm{~d}$ ).

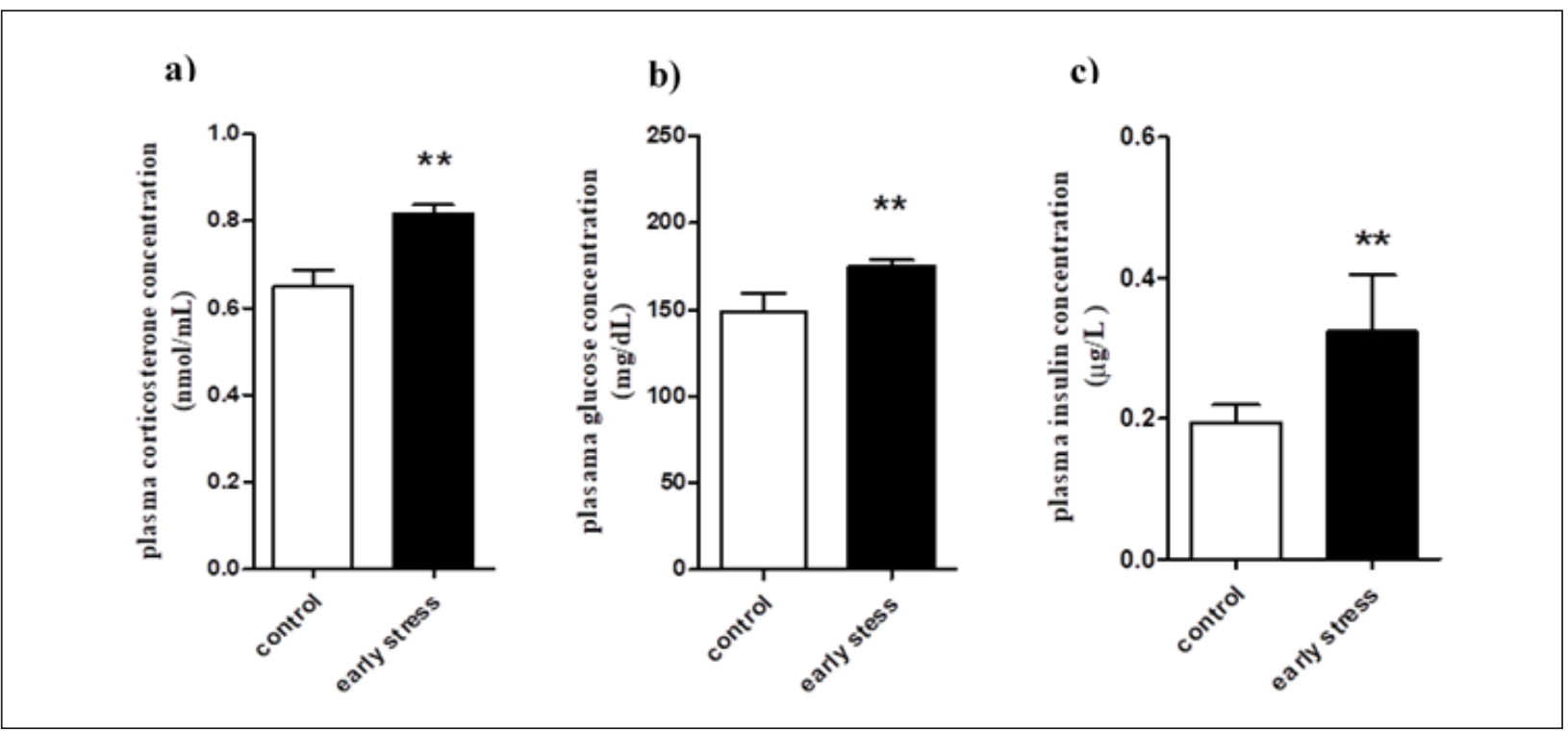

Figure1. Effect of postnatal foot shock stress on plasma corticosterone (a), plasma glucose (b), and plasma insulin (c) in rat pups. Control: non-foot shock stress, early stress: postnatal foot shock stress. Values are expressed as mean \pm SEM $(n=8)$. ${ }^{* *} p<0.01$ vs. control group. 


\section{Discussion}

The results of this study showed increased levels of corticosterone, insulin, and glucose following the induction of foot shock stress at early postnatal days 14-18. Furthermore, early postnatal stress reduced glucose tolerance in the early stress group later in young adulthood age as compared to the control group.

Several studies have shown that induction of early life stress, as different experimental models, can modulate the HPA axis activity. It seems that HPA activity depends on the duration of stress exposure. In the present study, foot shock stress applied twice a day for five consecutive days increased the level of plasma corticosterone as an index of stress, in comparison with the control group. However, the increase in corticosterone levels in the young adult rats, which experienced early life stress, did not sustain, although there was still a tendency to increase. Different models of postnatal stress studies, such as maternal deprivation or separation, had different effects on plasma corticosterone concentration of adulthood. For instance, Koe et al. (2014) did not observe changes in the plasma level of corticosterone in the adult male Wistar rats that experienced maternal separation stress on postnatal days 2-14. However, Maghami et al. (2017) and another similar study (Daniels et al. 2009) have exhibited that maternal separation for the first 53 days after birth significantly increased the basal concentration of plasma corticosterone in comparison to the non-stressed group. Stress in early life, as a critical period of organs development, can produce sustained changes in the HPA axis activity. Different experiments have shown increase (Daniels et al. 2009), decrease (Veenema et al. 2008) or no significant changes in basal corticosterone levels (Koe et al. 2014) due to early life stress. The reasons for these differences could be due to a variety of stress models, race, sex and age of animals, and the duration of stress exposure (Maghami et al. 2017). Elevated plasma corticosterone following the foot shock stress at this study may be due to reduced glucocorticoid receptors in the hippocampus, resulting in a decrease in the negative feedback function (Maghami et al. 2017). It could also be due to an increased the HPA axis activation, which in turn stimulates adrenal gland to release corticosterone (Uys et al. 2006; Korosi et al. 2010; Eiland and McEwen 2012; Bijani et al. 2013). In this study, early postnatal stress induced corticosterone hyperglycemia and consequently insulin secretion. Corticosterone induces hyperglycemia via the high activity of key enzymes involved in the hepatic gluconeogenesis and also reduction of glucose utilization in the peripheral tissues, such as the adipose

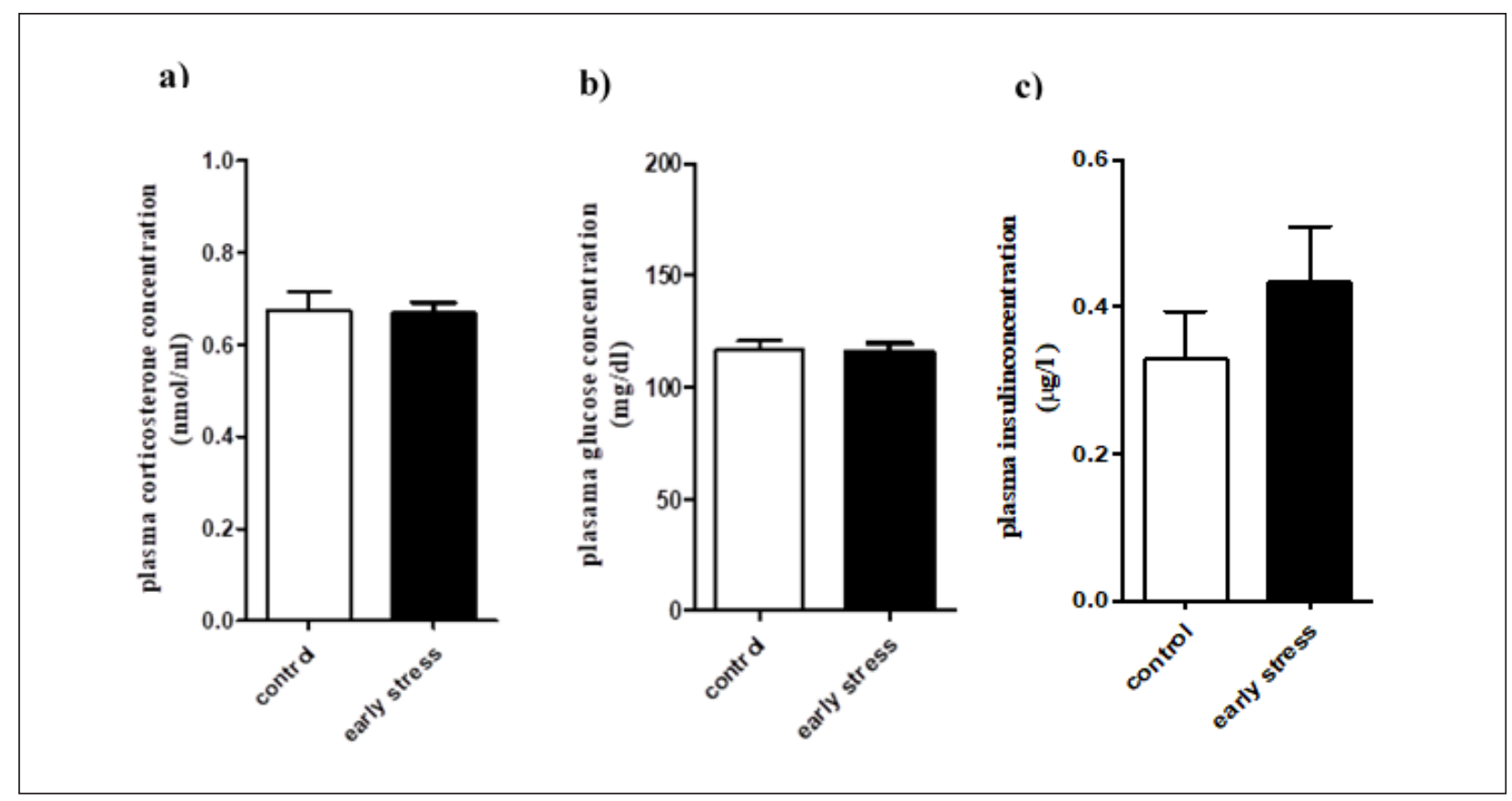

Figure 2. Effect of postnatal foot shock on fasting plasma corticosterone (a), plasma glucose (b), and plasma insulin (c) in young adult rats. Control: non-foot shock stress, early stress: postnatal foot shock stress. Values are expressed as mean \pm SEM $(n=8)$. 
a)

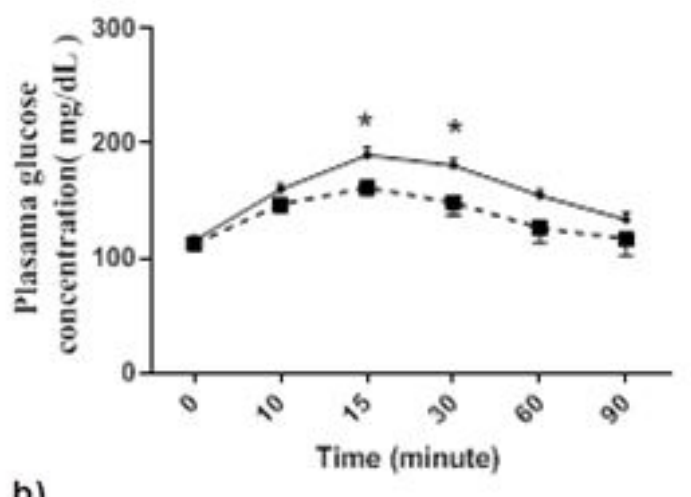

b)

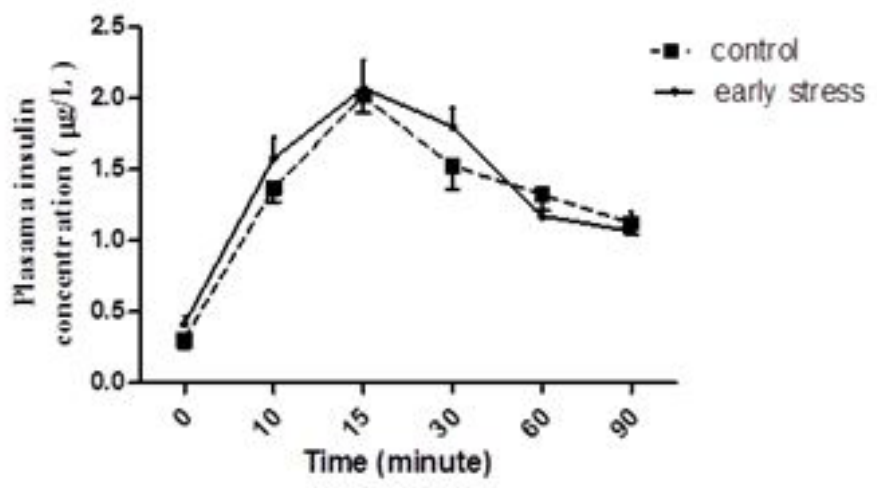

c)

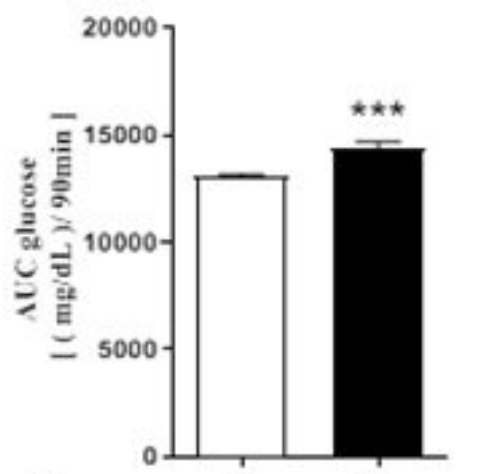

d)

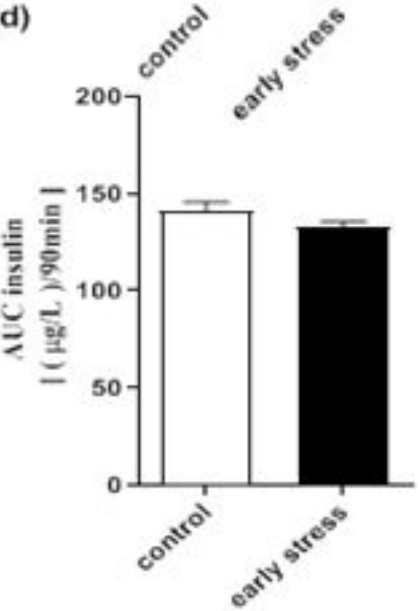

Figure 3. Changes in plasma glucose (a) and insulin concentration (b) or AUC glucose (c) and AUC insulin (d) during intraperitoneal glucose tolerance test (IPGTT) performance in young adult rats. Control: non-foot shock stress, early stress: postnatal foot shock stress. Values are expressed as mean $\pm \operatorname{SEM}(n=8) .{ }^{*} \mathrm{p}<0.05 ;{ }^{* *} \mathrm{p}<0.001$ vs. control group.

cells or skeletal muscles (inducing insulin resistance). In response to hyperglycemia, pancreas enhanced the insulin secretion from $\beta$-cells (Dungan et al. 2009; Marik and Bellomo 2013).

In the present study, in response to postnatal stress, significant increase in plasma glucose and insulin levels were observed, but later in young adulthood age, these markers return to the baseline. It has been proposed that the HPA axis recovery or adaptation following the early life stress, may be the cause of no significant increase in corticosterone and in turn glucose and insulin levels in young adulthood (Maniam et al. 2014). While maternal separation stress could increase the basal glucose in adolescence, it doesn't cause a significant change in plasma insulin (Maghami et al. 2017). In another chronic maternal separation stress, from the 3 to $7^{\text {th }}$ day after birth, early stress did not cause a significant change in plasma glucose and insulin in mature rats (McPherson et al. 2009). On the other hand, application of this stress for a 21-day period caused the insulin decrease in newborn rats (Solas et al. 2010). Using different species and various study design could be the cause of the discrepancy occurred among the results obtained in different studies.

Intraperitoneal glucose tolerance test was performed to evaluate postnatal stress in the development of insulin resistance during adulthood. Our results showed that foot shock stress for 5 consecutive days during the postnatal period could impair the glucose tolerance in the young adult rats. The glucose level and the insulin secretion at different times after i.p. injection of glucose were significantly higher in the early stress group than in the control group. Hyperglycemia and hyperinsulinemia are the primary symptoms of stress-induced insulin resis- 
tance (Singhal et al. 2018). Glucocorticoids are the main stress hormones, which can cause or contribute to insulin resistance. Glucocorticoids enhance oxidative stress-induced pre-inflammatory adipokines, such as tumor necrosis factor- $\alpha$ (TNF- $\alpha$ ) and interleukin-6 (IL-6), which can disrupt the signaling pathways of insulin-sensitive tissues and produce insulin resistance (Eguchi et al. 2011; Tangvarasittichai 2015; Singhal et al. 2018).

Late gestation and early postnatal times are critical periods during the normal development of many organs, including pancreas and insulin-sensitive tissues. Hence, any intervention in this period, such as stressful experiences or adverse conditions, can contribute to susceptibility of metabolic disorders such as type-2 diabetes later in life as a result of insulin resistance (McPherson et al. 2009). It has been reported in numerous studies that human and animal exposure to glucocorticoids during the critical period of the development of internal organs may lead to a glucose intolerance phenotype in adulthood. For example, reduced insulin synthesis and reduced mRNA levels of glucose transporter-2 (GLUT-2) as a key glucose sensor of pancreatic $\beta$-cells were generated by the chronic exposure to dexamethasone (Sadeghimahalli et al. 2015; Ghazalli et al. 2018). In fact, glucocorticoids cause glucose intolerance in adult offspring by inducing a permanent change in development of $\beta$-cells, which indicates the effects of metabolic programming of stress during these critical time periods (before and after birth) (Drake et al. 2007). Increased insulin secretion is the first sign of insulin resistance (Dancause et al. 2013) that results in increased insulin requirement in the early stages of insulin resistance, which enhances the insulin secretory capacity of pancreatic $\beta$-cells as a compensatory response (Weiss and Hagman 2018). Enhanced insulin secretion in response to i.p. injection of glucose in the present study suggested that postnatal stress was the reason for insulin resistance during adulthood. There was an increased risk of type- 1 diabetes in the children who had a high-tension life in the first two years of life or those who have experienced an apparent life-threatening event. Childhood abuse experiences were correlated with the elevated levels of adult pre-inflammatory cytokine (Lundgren et al. 2018). It seems that postnatal stress-induced disturbances of the HPA axis results in stable changes in the structure and function of the pancreas and insulin-sensitive tissues, such as the function of insulin receptors and muscle glucocorticoids receptors, which in turn leads to an insulin resistance. Furthermore, exposure to stress may trigger epigenetic changes in genes involving in insulin-secreted pancreatic $\beta$-cells that results in high insulin secretory capacity in response to insulin resistance (Cottrell and Seckl 2009). The nature of postpartum stressors probably determines different phenotypes observed in metabolic diseases.

In summary, the results of the present study indicate that increasing activity of the HPA axis, due to the postnatal stress, can interfere with the insulin secretion and glucose intolerance during adolescence, and thus can cause type- 2 diabetes phenotype in adulthood. Further studies are needed to explore the mechanisms by which the postnatal stress induces insulin resistance in adulthood. One of the limitations of this study was the lack of measurement of insulin and glucocorticoid receptors, oxidative stress, and pro-inflammatory mediators in adipose tissue, skeletal muscle or liver tissue, which deserves further studies.

\section{Acknowledgments}

This study was supported by a grant from the Research Deputy of Faculty of Medicine, Shahid Beheshti University of Medical Sciences.

\section{References}

Bijani S, Najafi Abedi A, Ranjbaran M, Sadeghi Gharajeh Daghi S, Ghoshooni H, Zardooz H, Bourbour Z, Sahraei H. Effects of noise pollution stress during pregnancy on anatomical and functional brain cortex development of the offsprings of NMRI mice. Koomesh 14, 192-199, 2013.

Bonner-Weir S. Perspective: postnatal pancreatic beta cell growth. Endocrinology 141, 1926-1929, 2000.

Chida Y, Sudo N, Sonoda J, Hiramoto T, Kubo C. Early-life psychological stress exacerbates adult mouse asthma via the hypothalamus-pituitary-adrenal axis. Am J Respir Crit Care Med 175, 316-322, 2007.

Cottrell EC, Seckl JR. Prenatal stress, glucocorticoids and the programming of adult disease. Front Behav Neurosci 3, 19, 2009.

Dancause KN, Veru F, Andersen RE, Laplante DP, King S. Prenatal stress due to a natural disaster predicts insulin secretion in adolescence. Early Hum Dev 89, 773-776, 2013. 
Daniels WM, Fairbairn LR, van Tilburg G, McEvoy CR, Zigmond MJ, Russell VA, Stein DJ. Maternal separation alters nerve growth factor and corticosterone levels but not the DNA methylation status of the exon 1(7) glucocorticoid receptor promoter region. Metab Brain Dis 24, 615-627, 2009.

Drake AJ, Tang JI, Nyirenda MJ. Mechanisms underlying the role of glucocorticoids in the early life programming of adult disease. Clin Sci 113, 219-232, 2007.

Dungan KM, Braithwaite SS, Preiser JC. Stress hyperglycaemia. Lancet 373, 1798-1807, 2009.

Eguchi R, Scarmagnani FR, Cunha CA, Souza GI, Pisani LP, Ribeiro EB, do Nascimento CMO, Spadari-Bratfisch RC, Oyama LM. Fish oil consumption prevents glucose intolerance and hypercorticosteronemy in footshockstressed rats. Lipids Health Dis 10, 71, 2011.

Eiland L, McEwen BS. Early life stress followed by subsequent adult chronic stress potentiates anxiety and blunts hippocampal structural remodeling. Hippocampus 22, 82-91, 2012.

Fowden AL, Hill DJ. Intra-uterine programming of the endocrine pancreas. Br Med Bull 60, 123-142, 2001.

Ghazalli N, Wu X, Walker S, Trieu N, Hsin LY, Choe J, Chen C, Hsu J, LeBon J, Kozlowski MT, Rawson J, Tirrell DA, Yip MLR, Ku HT. Glucocorticoid signaling enhances expression of glucose-sensing molecules in immature pancreatic beta-like cells derived from murine embryonic stem cells in vitro. Stem Cells Dev 27, 898-909, 2018.

Gluckman PD, Hanson MA, Cooper C, Thornburg KL. Effect of in utero and early-life conditions on adult health and disease. N Engl J Med 359, 61-73, 2008.

Hellerstrom C, Swenne I. Functional maturation and proliferation of fetal pancreatic $\beta$-cells. Diabetes 40, 89-93, 1991.

Hill DJ, Strutt B, Arany E, Zaina S, Coukell S, Graham CF. Increased and persistent circulating insulin-like growth factor II in neonatal transgenic mice suppresses developmental apoptosis in the pancreatic islets. Endocrinology 141, 1151-1157, 2000.

Jellyman JK, Allen VL, Holdstock NB, Fowden AL. Glucocorticoid overexposure in neonatal life alters pancreatic beta-cell function in newborn foals. J Anim Sci 91, 104-110, 2013.

Kaufman D, Banerji MA, Shorman I, Smith EL, Coplan JD, Rosenblum LA, Kral JG. Early-life stress and the development of obesity and insulin resistance in juvenile bonnet macaques. Diabetes 56, 1382-1386, 2007.

Koe AS, Salzberg MR, Morris MJ, O’Brien TJ, Jones NC. Early life maternal separation stress augmentation of limbic epileptogenesis: the role of corticosterone and HPA axis programming. Psychoneuroendocrinology 42, 124-133, 2014.

Korosi A, Shanabrough M, McClelland S, Liu ZW, Borok E, Gao XB, Horvath TL, Baram TZ. Early-life experience reduces excitation to stress-responsive hypothalamic neurons and reprograms the expression of corticotropin-releasing hormone. J Neurosci 30, 703-713, 2010.Loizzo S, Campana G, Vella S, Fortuna A, Galietta G, Guarino I, Costa L, Capasso A, Renzi P, Frajese GV. Post-natal stress-induced endocrine and metabolic alterations in mice at adulthood involve different pro-opiomelanocortin-derived peptides. Peptides 31, 2123-2129, 2010.

Lundgren M, Ellstrom K, Elding Larsson H; DiPiS study group. Influence of early-life parental severe life events on the risk of type 1 diabetes in children: the DiPiS study. Acta Diabetol 55, 797-804, 2018.

Maghami S, Sadeghimahalli F, Zardooz H. Effects of maternal separation stress on glucose homeostasis in pubertal male rats. Koomesh 19, 887-893, 2017.

Maniam J, Antoniadis C, Morris MJ. Early-life stress, HPA axis adaptation, and mechanisms contributing to later health outcomes. Front Endocrinol (Lausanne) 5, 73, 2014.

Marais L, Van Rensburg SJ, Van Zyl JM, Stein DJ, Daniels WM. Maternal separation of rat pups increases the risk of developing depressive-like behavior after subsequent chronic stress by altering corticosterone and neurotrophin levels in the hippocampus. Neurosci Res 61, 106-112, 2008.

Marik PE, Bellomo R. Stress hyperglycemia: an essential survival response! Crit Care 17, 305, 2013.

McPherson RJ, Mascher-Denen M, Juul SE. Postnatal stress produces hyperglycemia in adult rats exposed to hypoxia-ischemia. Pediatr Res 66, 278-282, 2009.

Sadeghimahalli F, Karbaschi R, Zardooz H, Khodagholi F, Rostamkhani F. Effect of early life stress on pancreatic isolated islets' insulin secretion in young adult male rats subjected to chronic stress. Endocrine 48, 493-503, 2015.

Singhal A, Agrawal A, Ling J. Regulation of insulin resistance and type II diabetes by hepatitis C virus infection: A driver function of circulating mi RNA s. J Cell Mol Med 22, 2071-2085, 2018.

Solas M, Aisa B, Mugueta MC, Del Rio J, Tordera RM, Ramirez MJ. Interactions between age, stress and insulin on cognition: implications for Alzheimer's disease. Neuropsychopharmacology 35, 1664-1673, 2010. 
Srinivasan M, Patel MS. Metabolic programming in the immediate postnatal period. Trends Endocrinol Metab 19, 146-152, 2008.

Tangvarasittichai S. Oxidative stress, insulin resistance, dyslipidemia and type 2 diabetes mellitus. World J Diabetes 6, 456-480, 2015.

Taylor SE. Mechanisms linking early life stress to adult health outcomes. Proc Natl Acad Sci U S A 107, 8507-8512, 2010.

Trombini M, Hulshof H, Graiani G, Carnevali L, Meerlo P, Quaini F, Sgoifo A. Early maternal separation has mild effects on cardiac autonomic balance and heart structure in adult male rats. Stress 15, 457-470, 2012.

Uys JD, Muller CJ, Marais L, Harvey BH, Stein DJ, Daniels WM. Early life trauma decreases glucocorticoid receptors in rat dentate gyrus upon adult re-stress: reversal by escitalopram. Neuroscience 137, 619-625, 2006.

Veenema AH, Reber SO, Selch S, Obermeier F, Neumann ID. Early life stress enhances the vulnerability to chronic psychosocial stress and experimental colitis in adult mice. Endocrinology 149, 2727-2736, 2008.

Weiss R, Hagman E. Pathogenesis of insulin resistance and glucose intolerance in childhood obesity. In: Freemark M. (eds) Pediatric Obesity. Contemporary Endocrinology. Humana Press, Cham, pp. 379-391, 2018.

Zeugmann S, Quante A, Popova-Zeugmann L, Kossler W, Heuser I, Anghelescu I. Pathways linking early life stress, metabolic syndrome, and the inflammatory marker fibrinogen in depressed inpatients. Psychiatr Danub 24, $57-65,2012$. 